\title{
A Case of Post-infectious Disseminated large sized multiple Pneumatoceles secondary to Staphylococcal sepsis
}

\author{
Rabindran ${ }^{1}$, Parakh $\mathbf{H}^{2}$ \\ ${ }^{1}$ Dr. Rabindran, Junior Consultant Neonatologist, ${ }^{2}$ Dr. Hemant Parakh, Consultant Neonatologist, Sunrise Superspeciality \\ Children's Hospital, Hyderabad, Andhra Pradesh, India
}

Address for correspondence: Dr Rabindran, E mail: rabindranindia@yahoo.co.in

\begin{abstract}
Pulmonary pneumatoceles are thin-walled, air-filled cysts that develop within the lung parenchyma. A 2 month old baby girl was admitted with Staphylococcal skin infection of the right arm. Incision \& drainage with Fasciotomy was done. On day 4 of admission, baby had progressive respiratory distress. Initial X-ray was suggestive of pneumothorax. Blood culture yielded methicillin resistant staphylococcus aureus. In view of clinical worsening inspite of antibiotic cover, CT scan was done which revealed multiple pneumatocele with bilateral lung cysts. This report emphasizes the importance of considering postinfectious pneumatoceles in the differential diagnosis in children with progressive respiratory distress with acute deterioration.
\end{abstract}

Keywords: Pulmonary Pneumatocele, Staphylococcal Sepsis, Postinfectious Pneumatocele

Manuscript received: $5^{\text {th }}$ Dec 2014, Reviewed: $11^{\text {th }}$ Dec 2014, Author Corrected: $19^{\text {th }}$ Jan 2014, Accepted for Publication: $11^{\text {th }}$ Feb 2015

\section{Introduction}

Staphylococcus aureus is a major cause of infection in infants \& Children [1]. Staphylococcal pneumonia is a serious and rapidly progressive infection associated with prolonged morbidity and high mortality [2]. Postinfectious pneumatocele occurs in $2-8 \%$ of all cases of pneumonia in children [3]. However, the frequency can be as high as $85 \%$ in staphylococcal pneumonias. Pulmonary pneumatoceles are thin-walled, air-filled cysts that develop within the lung parenchyma. They can be single emphysematous lesions but are more often multiple, thin-walled, air-filled, cystlike cavities. Limited data are available about infective pulmonary cysts in infants. We report a case of an infant, who developed multiple pneumatoceles after Staphylococcus aureus sepsis.

\section{Case Report}

A 2 month old baby girl was admitted to our hospital with history of swelling over the right upper limb noted over 2 days, associated with history of antecedent fever since 4 days. The infant was a singleton, born by cesarean section to a 25 -year-old mother after full term gestation. On examination there was tense swelling in the right upper limb. The overlying skin was tense \& shining. A soft spot was noted over the posterio- medial aspect of lower third of humerus. The distal vascularisation was good. The baby was started on intravenous antibiotics along with oral analgesics. A clinical impression of septic arthritis was made and $5 \mathrm{ml}$ of pus was aspirated and was sent for culture. X-Ray limb revealed no bony lesions. At this stage clinical suspicion of pyomyositis with impending compartmental syndrome was made and Incision \& drainage was planned.

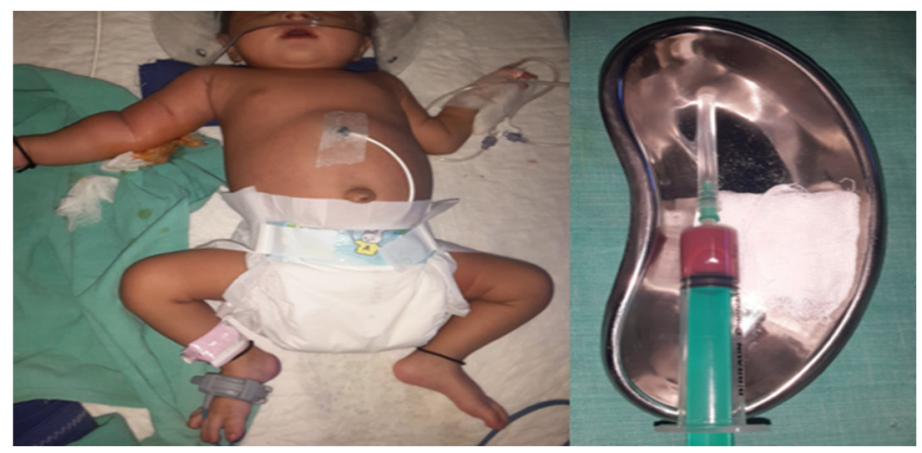

Figure- 1 : Left: Baby on Admission Right: Pus Aspirated

Incision \& drainage with Fasciotomy was done and 15-20 ml of pus was removed. Hemoglobin was $7.6 \mathrm{~g} / \mathrm{dl}$ and Packed Red Blood Cell transfusion was given. On day 4 of admission, baby had progressive respiratory distress with desaturation \& 
tachycardia developed abruptly. The findings on a initial radiograph of the chest were interpreted as indicating a pneumothorax, although a subsequent review of further X-rays showed cystic changes in both the lung fields.In view of progressive respiratory distress, she was started on assisted ventilation. A needle aspiration of air was performed, and a chest tube was inserted on emergency basis and water seal suctioning was instituted. Inotropes was started. Blood culture sent at the time of admission yielded methicillin resistant staphylococcus aureus. On day $6^{\text {th }}$ of admission, there was increase in C Reactive Protein levels $(90 \mathrm{mg} / \mathrm{L})$ with increase in White Blood Cell Counts (39000/cumm). The clinical conditions worsened dramatically \& the infant developed progressive respiratory insufficiency with severe hypercapnia and hypoxemia. The tachypnea increased with intercostal retractions, despite a functioning chest tube. Another ICD was placed, water seal suctioning was instituted.

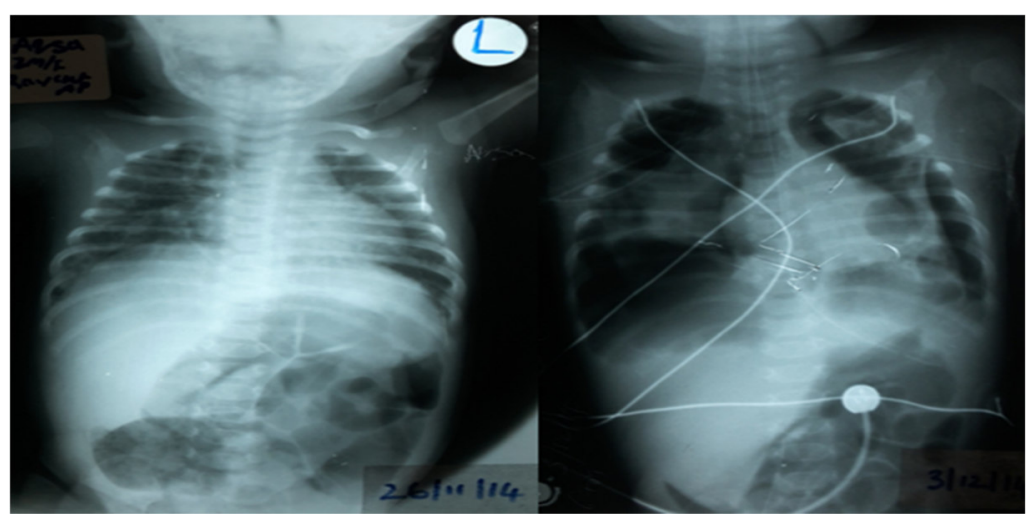

Figure-2: Left initial X-ray on admission, Right: X-ray a week later showing pneumatoceles \& bilateral pneumothorax

Repeat chest radiograph revealed diffuse bilateral interstitial and alveolar infiltrates, with multiple cystic areas and linear opacities distributed diffusely throughout both the lung fields. A small amount of parenchyma was visible and the heart size appeared normal. Intravenous antibiotics were continued along with total parenteral nutrition. Periodic Xrays were done which revealed poor lung expansion and multiple cystic areas. Computed tomographic (CT) scan of the thorax was done which revealed nearly total replacement of both lungs by multiple cysts, with areas of consolidation $\&$ collapse. The central airways appeared to be patent. Despite a maximum ventilator support, symptoms of respiratory distress did not improve and the baby could not be saved.

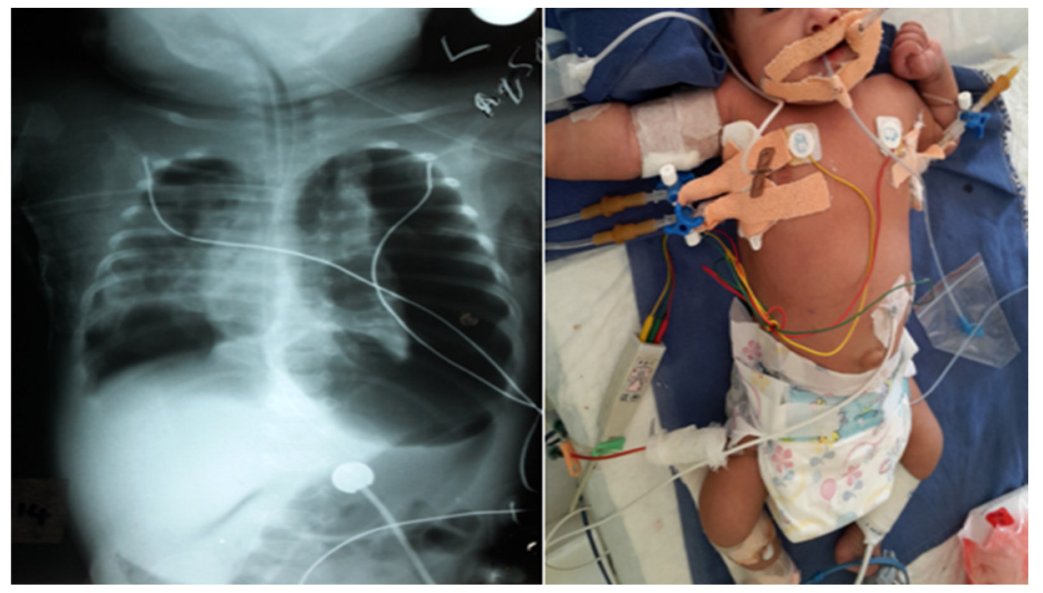

Figure-3 : Left: Recurrent Pneumothorax Right : Baby With Multiple Intercostal Drainage Tubes In Situ 


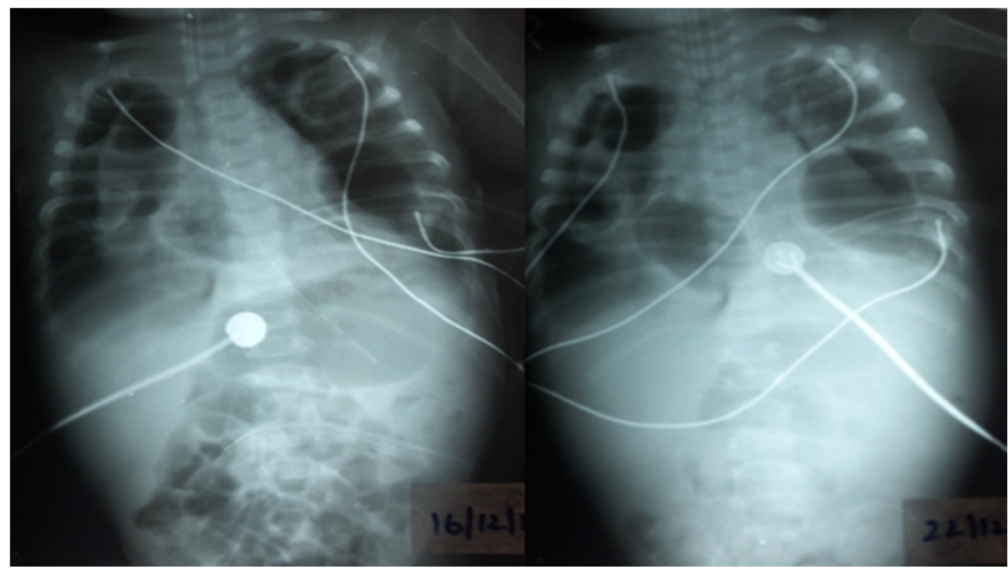

Case Report

Figure -4 : left: bilateral disseminated large sized pneumatoceles

Right: X- ray a week later showing persistent pneumatoceles

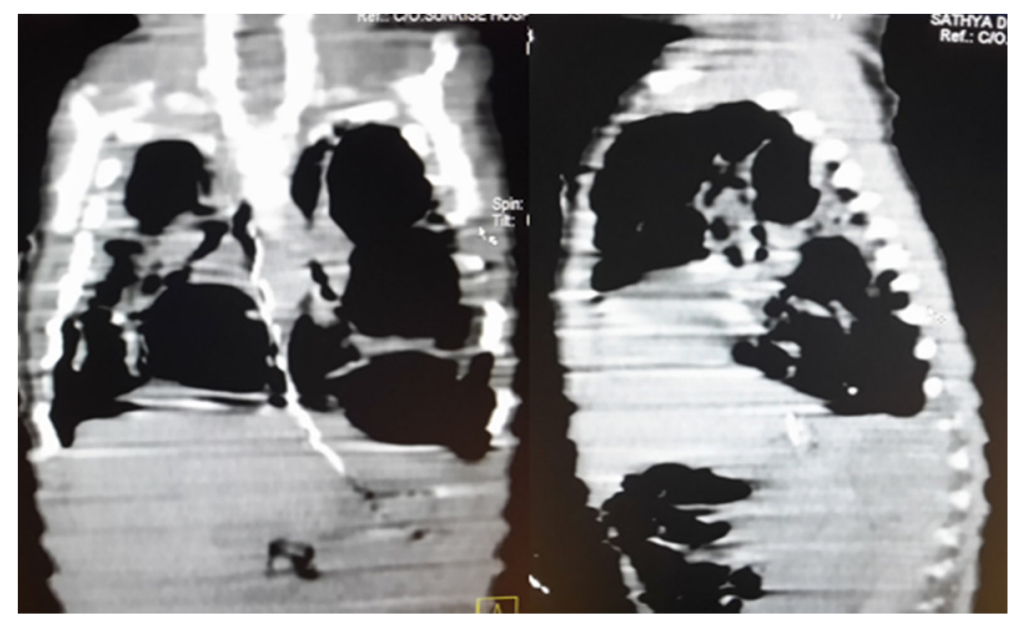

Figure- 5 : CT Scan Chest- Left: Coronal View Right: Saggital View

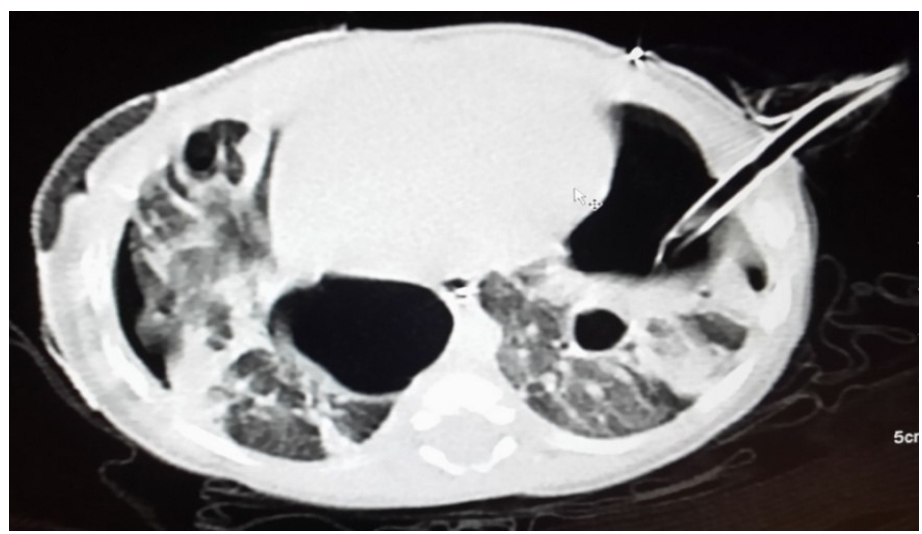

Figure-6 : Lung window axial image showing bilateral pneumothorax with intercostal drainage tube. Underlying lung show ground glass opacitis with multiple thin walled air filled pneumatoceles

\section{Differential Diagnosis}

Literature search for such a condition showed that Congenital and acquired conditions such as pulmonary sequestration, bronchogenic cyst, congenital lobar emphysema, congenital cystic adenomatoid malformation (CCAM), aspiration or bacterial pneumonia with cavitation, postinfectious pneumatoceles and Wilson- 
Mikity syndrome should be considered in the differential diagnosis of cystic pulmonary lesions in infants.

\section{Discussion}

Staphylococcus can cause furuncles, carbuncles, osteomyelitis, septic arthritis, wound infection, abscesses, pneumonia, empyema, endocarditis, pericarditis, meningitis and toxin-mediated diseases The pulmonary lesions caused by Staphylococcus aureus include localized or diffuse bronchopneumonia, lobar disease, dense consolidation, pneumatocele formation, pleural effusion, empyema, necrotizing pneumonitis, pneumothorax, pyopneumothorax and bronchopleural fistulas [1]. Pulmonary pneumatoceles are thin-walled, single or multiple emphysematous cystic lesions that develop within the lung parenchyma, as a sequelae to acute pneumonia, commonly caused by Staphylococcus aureus $[3,4]$ but also with other agents, including Streptococcus pneumoniae[2], Haemophilus influenzae, Escherichia coli, group A streptococci, Serratia marcescens, Klebsiella pneumoniae, adenovirus, \& tuberculosis $[5,6,7,8]$. Majority of pneumatoceles are asymptomatic and do not require surgical intervention[6]. Treatment of the underlying pneumonia with antibiotics is the first-line therapy. Postinfectious pneumatocele occurs in $2-8 \%$ of all cases of pneumonia in children [3]. However, the frequency can be as high as $85 \%$ in staphylococcal pneumonias. Children less than 1 year account for three fourths of all cases of staphylococcal pneumonia. Kunyoshi et al.[9] reported that $70 \%$ of pneumatoceles occurred in children younger than 3 years. Children usually present with typical features of pneumonia, including cough, fever, and respiratory distress and it is difficult to differentiate pneumonia with or without pneumatocele formation. Initial chest radiography often reveals pneumonia without evidence of a pneumatocele. Radiographic evidence of a pneumatocele most often occurs on day 5-7 of hospitalization as in our case.

Our objective is to discuss the nature of staphylococcal disease and its potential to cause serious complications. Awareness of such complications will result in providing prompt management.This report emphasizes the importance of considering postinfectious pneumatoceles in the differential diagnosis in children with progressive respiratory distress with acute deterioration.

\section{References}

1. Prince AS. Staphylococcal infections: Katz SL, Gershon AA, Hotez PJ. Infectious Diseases of Children, 10th edition. CV Mosby Company, St Louis, 1998; 47486.

2. Joosten KF, Hazelzet JA, Tiddens H. Staphylococcal pneumonia in childhood: will early surgical intervention lower mortality? Pediatr Pulmonol 1995, 20(2):83-8.

3. Amitai I, Mogle P, Godfrey S, Aviad I. Pneumatocele in infants and children. Report of 12 cases. Clin Pediatr. June 1983;22(6):420-2.

4. Dines DA: Diagnostic significances of pneumonia of the lung. JAMA 1968;204:1169-1182

5. Hussain N, Noce T, Sharma P, Jagjivan B, Hegde P, Pappagallo M. Pneumatoceles in preterm infantsincidence and outcome in the post-surfactant era. J Perinatol. May 2010;30(5):330-6.

6. Arora P, Kalra VK, Natarajan G. Pneumatoceles in infants in the neonatal intensive care unit: Clinical characteristics and outcomes. Am J Perinatol; 2013 Sep;30(8):689-94

7. Papageougiou A, Bauer CR, Fletcher BD, Stern L. Klebsiella pneumonia with pneumatocele formation in newborn infant. Can Med Assoc J 1973;109:1217-1219.

8. Bermejo Velasco E, Gonza'lez Molina E, Martinez Ayucar M, Zubia Aguillo A, Nieves Gil A, Salado Marin C, Diez Orive M. Pneumatocele as a complication of E. coli pneumonia in a newborn infant. Anales Espanoles de Pediatria 1992;37:526-528.

9. Kunyoshi V, Cataneo DC, Cataneo AJ. Complicated pneumonias with empyema and/or pneumatocele in children. Pediatr Surg Int 2006;22(2):186-90. 40.

\section{How to cite this article?}

Rabindran, Parakh H. A Case of Post-infectious Disseminated large sized multiple Pneumatoceles secondary to Staphylococcal sepsis. Pediatr Rev: Int J Pediatr Res 2014;1(3):83-86. doi: 10.17511/ijpr.2014.i03.04 